\title{
Effective Theories of Coupled Classical and Quantum Variables
}

\author{
from Decoherent Histories:
}

\section{A New Approach to the Backreaction Problem}

\author{
J. J. HALLIWELL ${ }^{\dagger}$ \\ Theory Group, Blackett Laboratory \\ Imperial College, London SW7 2BZ \\ $U K$ \\ Preprint IC 96-97/45, quant-ph/9705005. May, 1997 \\ Submitted to Physical Review D
}

\begin{abstract}
:
We use the decoherent histories approach to quantum theory to derive the form of an effective theory describing the coupling of classical and quantum variables. The derivation is carried out for a system consisting of a large particle coupled to a small particle with the important additional feature that the large particle is also coupled to a thermal environment producing the decoherence necessary for classicality. The effective theory is obtained by tracing out both the environment and the small particle variables. It consists of a formula for the probabilities of a set of histories of the large particle, and depends on the dynamics and initial quantum state of the small particle. It has the form of an almost classical particle coupled to a stochastic variable whose probabilities are determined by a formula very similar to that given by quantum measurement theory for continuous measurements of the small particle's position. The effective theory gives intuitively sensible answers when the small particle is in a superposition of localized states (unlike the simple mean field approach of coupling to the expectation values of the small system). The derived effective theory suggests a form of the semiclassical theory even when the quantum theory of the large system is not known, as is the case, for example, when a classical gravitational field is coupled to a quantized matter field, thus offerering a new approach to the backreaction problem.
\end{abstract}

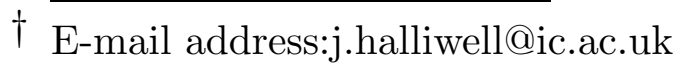




\section{INTRODUCTION}

What happens when a classical system interacts with a quantum system in a non-trivial superposition state? Quantum field theory in curved spacetime is an example of a number of situations where one would like to know the answer this question. There, the effect of the quantized matter field on the classical gravitational field is often assessed using the semiclassical Einstein equations [1,2]:

$$
G_{\mu \nu}=8 \pi G\left\langle T_{\mu \nu}\right\rangle
$$

The left hand side is the Einstein tensor of the classical metric field $g_{\mu \nu}$ and the right hand side is the expectation value of the energy momentum tensor of a quantum field.

Although we do not yet have the complete, workable theory of quantum gravity required to derive an equation like (1.1), on general grounds it is clear that it is unlikely to be valid unless the fluctuations in $T_{\mu \nu}$ are small $[3,4,5]$. Indeed, (1.1) fails to give intuitively sensible results when the matter field is in a superposition of localized states $[6,7]$. It is by no means obvious, however, that we have to resort to quantum gravity to accommodate non-trivial matter states. This leads one to ask whether there exists a semiclassical theory with a much wider range of validity than (1.1), which gives intuitively reasonable results for non-trivial superposition states for the matter field.

The object of the present paper is to derive the form of an effective theory of coupled classical and quantum variables, in some simple models where the quantum theory of the entire system is known. From there, we can then make a reasonable postulate as to the form such a theory might take even when the quantum theory of the variables treated classically is not known. Of course, a number of previous authors have attempted either to derive or postulate the form of theories of coupled classical and quantum variables [8]. What is 
perhaps missing from most of these earlier approaches is an adequate characterization of what it means for one of the subsystems to be effectively classical. Here, we will work in the context of the decoherent histories approach to quantum theory $[9,10,11,12]$, where a thorough characterization of what it means to be classical has been undertaken. This issue is an involved one, but simply, the system must be described by a decoherent set of histories consisting of the same type of variables at each moment of time whose probabilities are strongly peaked about classical equations of motion.

It should be stressed that we do not expect to derive a consistent theory describing the coupling of fundamentally classical variables to quantum variables. Rather, we are looking for the form of an effective theory in which variables which are the classical descendents of a (perhaps unknown) quantum theory couple to quantum variables. In contrast to fundamentally classical variables, classical descendents of quantum variables always suffer a certain amount of imprecision, partly due to their quantum fluctuations, but largely as a result of the coarse graining required for decoherence and hence, to render them effectively classical. This imprecision feeds into the quantum variables they couple to, and, as we shall see, confers some useful features.

In this paper, we will concentrate on some simple models in non-relativistic quantum theory. To motivate the discussion, consider the following system. Suppose we have a large ("to be classical") particle with coordinates $X$ linearly coupled to a small particle with coordinates $x$. Let the action be

$$
S=\int d t\left(\frac{1}{2} M \dot{X}^{2}+\frac{1}{2} m \dot{x}^{2}-\frac{1}{2} m \omega^{2} x^{2}-\lambda X x\right)
$$

Hence the equations of motion are

$$
\begin{array}{r}
M \ddot{X}+\lambda x=0 \\
m \ddot{x}+m \omega^{2} x+\lambda X=0
\end{array}
$$


A naive semiclassical approach (the mean field approach), on which (1.1) is based, involves considering the equation

$$
M \ddot{X}+\lambda\langle x\rangle=0
$$

together with the Schrödinger equation for the state of the small system with $X(t)$ as an external classical source. However, as stated above we do not expect (1.5) to have a very wide range of validity.

Physically, when a large, classical particle interacts with a small quantum system, the large particle in some sense "measures" the position of the small system at each moment of time, and then evolves according to the measured value. The probability for the large particle to measure a particular value of $x$ will be determined by the quantum state of the small system and there will generally be non-zero probabilities for a wide range of different values of $x$. There is no reason why the average value, $\langle x\rangle$, is the one that will almost always be measured, unless the coupling is very weak, or the distribution of $x$ is strongly peaked about $\langle x\rangle$. Therefore, what we expect in general is an ensemble of trajectories for the large particle, with a probability for each trajectory determined by the quantum state of the small particle. In this paper, we will derive a scheme of this type, using the decoherent histories approach, in a class of simple models.

We mention in passing that it is possible to proceed differently from this point and directly write down a phenomenological scheme for the coupling of classical and quantum variables using continous quantum measurement theory $[13,14,15,16,17]$. Such an approach was considered in Ref.[18]. The idea is that in (1.3), $X$ is treated as a classical variable, and $x$ is replaced by a classical stochastic variable $\bar{x}(t)$, the probability for which is given by a standard construction of quantum measurement theory:

$$
p[\bar{x}(t)]=\int \mathcal{D} x \mathcal{D} y \quad \rho_{0}^{B}\left(x_{0}, y_{0}\right) \exp \left(-\int d t \frac{(x-\bar{x})^{2}}{2 \sigma_{1}^{2}}-\int d t \frac{(y-\bar{x})^{2}}{2 \sigma_{1}^{2}}\right)
$$




$$
\begin{aligned}
& \times \exp \left(\frac{i}{\hbar} \int d t\left(\frac{1}{2} m \dot{x}^{2}-\frac{1}{2} m \omega^{2} x^{2}-\lambda x X\right)\right) \\
& \times \exp \left(-\frac{i}{\hbar} \int d t\left(\frac{1}{2} m \dot{y}^{2}-\frac{1}{2} m \omega^{2} y^{2}-\lambda y X\right)\right)
\end{aligned}
$$

where $\rho_{0}^{B}\left(x_{0}, y_{0}\right)$ is the initial density matrix of the small quantum system. Therefore, the scheme is to solve the equations of motion for the large particle with $\bar{x}(t)$ regarded as a classical source, and then the probability distribution on the trajectories $X(t)$ is that implied by the probability (1.8).

The formula (1.8) contains an arbitrary parameter $\sigma_{1}$ representing the imprecision in the continous measurement. A reasonable estimate as to its value can be made by appealing to the fact that, as stated above, $X$ is not fundamentally classical but a classical descendent of a quantum variable. It therefore has intrinsic imprecision, which limits the precision with which it can carry out "measurements" of the small particle. The parameter $\sigma_{1}$ ought therefore to be approximately determined given the size of the fluctuations in $X$ and the nature of the coupling between $X$ and $x[18]$.

The scheme we derive in this paper turns out to be very closely related to the phenomenological scheme presented in Ref.[18] (although it is not exactly the same). Furthermore, it yields a definite value for the parameter $\sigma_{1}$.

We will use the decoherent histories approach to quantum theory $[9,10,11,12,19]$. In this approach, the primary focus is on the probabilities for a set of histories of a closed system:

$$
p(\alpha)=\operatorname{Tr}\left(P_{\alpha_{n}}\left(t_{n}\right) \cdots P_{\alpha_{2}}\left(t_{2}\right) P_{\alpha_{1}}\left(t_{1}\right) \rho P_{\alpha_{1}}\left(t_{1}\right) \cdots P_{\alpha_{n}}\left(t_{n}\right)\right)
$$

Here, the $P_{\alpha}(t)$ are projection operators in the Heisenberg picture,

$$
P_{\alpha}(t)=e^{\frac{i}{\hbar} H t} P_{\alpha} e^{-\frac{i}{\hbar} H t}
$$


They are exhaustive and exclusive, which means, respectively,

$$
\sum_{\alpha} P_{\alpha}=1, \quad P_{\alpha} P_{\beta}=\delta_{\alpha \beta} P_{\alpha}
$$

The projection operators describe the possible properties the system may have at each moment of time. In this paper we are mainly interested in histories characterized by imprecisely specified positions, in which case the projectors have the form,

$$
P_{\alpha}=\int_{\alpha} d x|x\rangle\langle x|
$$

where the integral is over some interval on the real axis labeled by $\alpha$. In practice it is often more convenient to work with so-called Gaussian projectors,

$$
P_{\bar{x}}=\frac{1}{\left(2 \pi \sigma^{2}\right)^{\frac{1}{2}}} \int_{-\infty}^{\infty} d x \exp \left(-\frac{(x-\bar{x})^{2}}{2 \sigma^{2}}\right)
$$

which are only approximately exclusive.

Probabilities generally cannot be assigned to sets of histories unless there is negligible interference between them. The measure of interference between any pair of histories is the decoherence functional,

$$
D\left(\alpha, \alpha^{\prime}\right)=\operatorname{Tr}\left(P_{\alpha_{n}}\left(t_{n}\right) \cdots P_{\alpha_{2}}\left(t_{2}\right) P_{\alpha_{1}}\left(t_{1}\right) \rho P_{\alpha_{1}^{\prime}}\left(t_{1}\right) \cdots P_{\alpha_{n}^{\prime}}\left(t_{n}\right)\right)
$$

When the condition of (approximate) decoherence is satisfied,

$$
D\left(\alpha, \alpha^{\prime}\right) \approx 0 \quad \text { for } \quad \alpha \neq \alpha^{\prime}
$$

the interference between histories is negligible and probabilities obeying the probability sum rules may be assigned using the formula (1.9). The decoherence condition is typically only satisfied for histories that are coarse grained, i.e., histories for which the projections ask only very limited questions. 
A number of recent papers have used the decoherent histories approach to discuss the emergence of classical behaviour in simple particle models $[10,20,21,22,23]$. The decoherent histories approach is perhaps the most useful approach to this problem primarily for the following reason. When we say, in the context of quantum theory, that a particle exhibits almost classical behaviour, we mean that the probability that it is found at a sequence of imprecisely specified positions at a sequence of times exists, and furthermore, that this probability is peaked about classical equations of motion $[24,10]$. Hence to talk about classical properties of a point particle, we need to talk about the histories of imprecisely specified positions.

A commonly used coarse graining procedure to ensure that histories of position are decoherent is to couple to a thermal environment. We therefore consider projections at each moment of time of the form

$$
P_{\alpha}=P_{\alpha}^{A} \otimes I^{\mathcal{E}}
$$

where $P_{\alpha}^{A}$ denotes imprecise position projections for the particle, and $I^{\mathcal{E}}$ denotes the identity on the environment. Using this basic set-up, a number of recent papers have shown that for a thermal environment of sufficiently high temperature, there exist decoherent histories of imprecisely specified position [10,23]. Furthermore, the probabilities for histories are then strongly peaked about classical equations of motion with dissipation, with thermal fluctuations about them $[10,21]$. If the particle is sufficiently massive, the effect of the thermal fluctuations is very small, and its behaviour may therefore be said to be effectively classical.

Given, therefore, this characterization of what it means for a particle to be effectively classical, we may now turn to the main question we are interested in, which is to determine the form of the effective equations of motion when the classical particle is coupled to a small quantum particle. It should be clear that it is very easy to set up this problem in 
the decoherent histories approach. We quite simply couple a small particle in an arbitrary initial state to the case considered above. The closed system we consider therefore consists of a large particle (A) coupled to a small particle (B). The large particle is also coupled to a thermal environment $(\mathcal{E})$. The projections at each moment of time are therefore of the form,

$$
P_{\alpha}=P_{\alpha}^{A} \otimes I^{B} \otimes I^{\mathcal{E}}
$$

where $I^{B}$ denotes the identity for the small particle. We again expect decoherence of the histories. The main thing we are interested in is the probability distribution for the histories of the large particle: we expect it to be similar to the case above, but modified in a way depending on the dynamics of the small particle and also its initial state (which we leave arbitrary).

Note that, since we are only interested in the effective equations of motion for the large particle, $A$, we do not consider projections onto the properties of the small particle, $B$. The histories of the small particle therefore do not need to be decoherent (indeed ,the interesting case is that in which they might exhibit quantum behaviour), and it is for this reason that we do not couple the small particle to the environment.

As we shall see, it is easy to set up the expression for the probabilitity for histories of the large particle. The main issue is to express the result in a useful and recognizable form. We shall show that the effective equations of motion have the form of the classical equations of motion coupling $X$ to $x$, but with the small particle variables $x$ replaced by a stochastic c-number $\bar{x}(t)$. Moreover, the probability distribution for $\bar{x}(t)$ is given by a formula bearing a close resemblance to the probability for a continuous position measurement in continuous quantum measurement theory.

The majority of our results are described in Section 2, where we consider the simple 
linear model described above, linearly coupled to a thermal environment. We compute the probabilities for histories of the large particle. It has the form of a stochastic theory in which a classical variable $X$ is coupled to a stochastic variable $\bar{x}(t)$ with a probability distribution for $\bar{x}(t)$. The distribution of $\bar{x}(t)$, in this simple linear model, essentially reduces to a Wigner function on the initial phase space data of the small particle (although smeared over a large region of phase space, so that it is positive). We discuss some properties of the scheme, and show that the naive semiclassical approximation is recovered in the limit of very weak coupling. We also show that if the small particle is initially in a superposition of localized states, the large particle "sees" one or other of the localized states, and not the mean position of the entire state.

In Section 3 we demonstrate the connection with quantum theory of continous measurements. We show that the probability distribution for $\bar{x}(t)$ is closely related to the formula for continuous quantum measurements, (1.8), and discuss the connection with the phenomenological scheme of Ref.[18].

The generalization to non-linear systems with non-trivial couplings is straightforward, and is considered in Section 4. Couplings involving the energy of the small particle in Section 5. We summarize and conclude in Section 6.

\section{A SIMPLE LINEAR MODEL}

We now compute the decoherence functional for a simple linear model. The model consists of a large free particle linearly coupled to a small harmonic oscillator (with action Eq.(1.2)), but the large particle is also coupled to a thermal bath. The large particle could start out in an arbitrary state, but we are assuming it is almost classical, so it is most 
useful to start it out in a state with almost definite position and momentum. The nearclassical behaviour of the large particle is assured by its coupling to the environment. The small particle starts out in an arbitrary initial state. We would like to know how the large classical particle responds to the presence of the small quantum particle in an arbitrary quantum state. More precisely, what is the effective description of the large particle, in terms of the quantum state of the small particle? In the decoherent histories approach we can quite simply calculate directly the probability that the large particle will take a particular trajectory.

\section{2(A). Probabilities for Histories}

After tracing out the thermal bath modes, the decoherence functional for the model is

$$
\begin{aligned}
D[\bar{X}, \bar{Y}]= & \int \mathcal{D} X \mathcal{D} Y \mathcal{D} x \mathcal{D} y \rho_{0}^{A}\left(X_{0}, Y_{0}\right) \rho_{0}^{B}\left(x_{0}, y_{0}\right) \\
& \times \exp \left(-\int d t \frac{(X-\bar{X})^{2}}{2 \sigma^{2}}-\int d t \frac{(Y-\bar{Y})^{2}}{2 \sigma^{2}}\right) \\
& \times \exp \left(\frac{i}{\hbar} \int d t\left(\frac{1}{2} M \dot{X}^{2}-\frac{1}{2} M \dot{Y}^{2}\right)-D \int d t(X-Y)^{2}\right) \\
& \times \exp \left(\frac{i}{\hbar} \int d t\left(\frac{1}{2} m \dot{x}^{2}-\frac{1}{2} m \omega^{2} x^{2}-\lambda X x\right)\right) \\
& \times \exp \left(-\frac{i}{\hbar} \int d t\left(\frac{1}{2} m \dot{y}^{2}-\frac{1}{2} m \omega^{2} y^{2}-\lambda Y y\right)\right)
\end{aligned}
$$

This formula is an elementary generalization of similar ones used in Refs.[10,25,21]. The integration is over paths $X(t), Y(t), x(t), y(t)$ which fold into the initial density matrices $\rho^{A}\left(X_{0}, Y_{0}\right), \rho^{B}\left(x_{0}, y_{0}\right)$ at the initial time, and at the final time, $X=Y$ and $x=y$ are integrated over. We have used Gaussian projections of width $\sigma$ to specify the trajectories of the large particle (although we did not need to do this - exact projections may have been used, but this is a bit more awkward [10]). The influence functional formalism of 
Feynman and Vernon has been used to handle the thermal bath [26,27]. The only remnant of this environment is the term proportional to $(X-Y)^{2}$, and the constant $D$ is given by $D=2 M \gamma k T / \hbar^{2}$. For simplicity we are working in the limit of high temperature and negligible dissipation, but these restrictions are easily relaxed.

For macroscopic values of $M, T$ and $\gamma, D$ is exceedingly large, thereby very effectively suppressing contributions from widely different values of $X$ and $Y$. The coarse graining scale of $X$ and $Y$ is set by the parameter $\sigma$, hence the condition for approximate decoherence is $D>1 / \sigma^{2}[23,25]$. We are generally interested in histories which are maximally refined, that is, as fine-grained as possible consistent with a given standard of approximate decoherence [10]. This means, in this case, that $\sigma$ is taken to be as small as possible, which means that it is of order $D^{-\frac{1}{2}}$.

The probabilities for histories $\bar{X}(t)$, which may now be assigned, are given by the diagonal elements of (2.1). Introducing

$$
Q=\frac{1}{2}(X+Y), \quad \xi_{1}=X-Y
$$

the integration over $\xi_{1}$ may be carried out, and the probabilities are

$$
\begin{aligned}
p[\bar{X}(t)]= & \int \mathcal{D} Q \mathcal{D} x \mathcal{D} y W_{0}^{A}\left(M \dot{Q}_{0}, Q_{0}\right) \rho^{B}\left(x_{0}, y_{0}\right) \\
& \times \exp \left(-\int d t \frac{(Q-\bar{X})^{2}}{\sigma^{2}}-\frac{1}{4 \hbar^{2} \tilde{D}} \int d t\left(M \ddot{Q}+\frac{1}{2} \lambda(x+y)\right)^{2}\right) \\
& \times \exp \left(\frac{i}{\hbar} \int d t\left(\frac{1}{2} m \dot{x}^{2}-\frac{1}{2} m \omega^{2} x^{2}-\lambda Q x\right)\right) \\
& \times \exp \left(-\frac{i}{\hbar} \int d t\left(\frac{1}{2} m \dot{y}^{2}-\frac{1}{2} m \omega^{2} y^{2}-\lambda Q y\right)\right)
\end{aligned}
$$

where $\tilde{D}=D+1 /\left(4 \sigma^{2}\right)$. An integration by parts was performed, in the exponent, which picks up a boundary term $-\frac{i}{\hbar} M \dot{Q}(0) \xi_{1}(0)$ (recall that $\xi_{1}=0$ at the final time). The integration over $\xi_{1}(0)$ then effectively produces the Wigner transform $W_{0}^{A}$ of the initial density matrix $\rho_{0}^{A}[10,28]$. 
Eq.(2.3) may be written,

$$
\begin{aligned}
p[\bar{X}(t)]= & \int \mathcal{D} \bar{q} \mathcal{D} Q W_{0}^{A}\left(M \dot{Q}_{0}, Q_{0}\right) w_{Q}[\bar{q}(t)] \\
& \times \exp \left(-\int d t \frac{(Q-\bar{X})^{2}}{\sigma^{2}}-\frac{1}{4 \hbar^{2} \tilde{D}(1-\eta)} \int d t(M \ddot{Q}+\lambda \bar{q})^{2}\right)
\end{aligned}
$$

where

$$
\begin{aligned}
w_{Q}[\bar{q}(t)]= & \int \mathcal{D} x \mathcal{D} y \rho_{0}^{B}\left(x_{0}, y_{0}\right) \exp \left(-\frac{\lambda^{2}}{4 \hbar^{2} \tilde{D} \eta} \int d t\left(\frac{(x+y)}{2}-\bar{q}\right)^{2}\right) \\
& \times \exp \left(\frac{i}{\hbar} \int d t\left(\frac{1}{2} m \dot{x}^{2}-\frac{1}{2} m \omega^{2} x^{2}-\lambda Q x\right)\right) \\
& \times \exp \left(-\frac{i}{\hbar} \int d t\left(\frac{1}{2} m \dot{y}^{2}-\frac{1}{2} m \omega^{2} y^{2}-\lambda Q y\right)\right)
\end{aligned}
$$

To achieve the decomposition (2.5) we have effectively deconvolved the second part of the Gaussian in Eq.(2.4), using the functional integral generalization of the formula,

$$
\exp \left(-(x-y)^{2}\right)=\int d z \exp \left(-\frac{(x-z)^{2}}{1-\eta}-\frac{(y-z)^{2}}{\eta}\right)
$$

This deconvolution is of course not unique, and $\eta$ is an arbitrary constant parametrizing this non-uniqueness (although clearly the total probability distribution (2.4) is independent of $\eta$ ). This trick turns out to be useful for smearing the Wigner functions of each particle, thereby rendering them positive $[29,28]$.

Written in the form (2.4) the probability distribution now has a reasonably natural interpretation. First of all recall that we are assuming that the Wigner function of the large particle is strongly peaked about particular values of $Q_{0}$ and $M \dot{Q}_{0}$. Hence in the absence of the coupling to the small particle, Eq.(2.4) describes a probability distribution for the large particle strongly peaked about a single classical solution with prescribed initial conditions. The width of peaking about the classical solution is controlled by the factor $\hbar^{2} \tilde{D}$, which is of order $M \gamma k T$, and this is typically very small for macroscopic values of $M, \gamma$ and $T$. 
With the small particle coupled in, however, there is the integration over $\bar{q}(t)$ together with the weight function (2.5). In the next section, we will show that it is closely related to the probabality distribution for continously measuring the position $q(t)$ of the small particle. Eq.(2.4) is therefore the sought-after result: it describes an ensemble of trajectories for the large particle with a weight depending on the initial conditions and dynamics of the small particle.

\section{2(B). The Weight Function}

The weight function (2.5) may be further evaluated as follows. Introduce $q=\frac{1}{2}(x+y)$ and $\xi_{2}=x-y$. Then the $\xi_{2}$ integral may be done with the result,

$$
\begin{aligned}
w_{Q}[\bar{q}(t)]= & \int \mathcal{D} q W_{0}^{B}\left(m \dot{q}_{0}, q_{0}\right) \exp \left(-\frac{\lambda^{2}}{4 \hbar^{2} \tilde{D} \eta} \int d t(q-\bar{q})^{2}\right) \\
& \times \delta\left[m \ddot{q}+m \omega^{2} q+\lambda Q\right]
\end{aligned}
$$

where $W_{O}^{B}$ is the initial Wigner function of the small particle. Now let

$$
q(t)=q_{0} \cos \omega t+\frac{\dot{q}_{0}}{\omega} \sin \omega t+\lambda \int d t^{\prime} G\left(t, t^{\prime}\right) Q\left(t^{\prime}\right)+\delta q(t)
$$

where $G\left(t, t^{\prime}\right)$ is the Green function for the harmonic oscillator, and $\delta q(0)=0=\delta \dot{q}(0)$. Then the delta functional in the functional integration in Eq.(2.7) becomes $\delta[m \delta \ddot{q}(t)]$, which implies, given the above initial conditions, that $\delta q(t)=0$. All that remain are two ordinary integrations over $q_{0}$ and $p_{0}=m \dot{q}_{0}$ :

$$
\begin{aligned}
w_{Q}[\bar{q}(t)]= & \int d p_{0} d q_{0} W_{0}^{B}\left(p_{0}, q_{0}\right) \\
& \times \exp \left(-\frac{\lambda^{2}}{4 \hbar^{2} \tilde{D} \eta} \int d t\left(q_{0} \cos \omega t+\frac{p_{0}}{m \omega} \sin \omega t+\lambda \int d t^{\prime} G\left(t, t^{\prime}\right) Q\left(t^{\prime}\right)-\bar{q}(t)\right)^{2}\right)
\end{aligned}
$$


This shows that the weight function is in fact a Wigner function smeared over a region of phase space of size $\Delta$, where

$$
\Delta \sim \frac{\hbar^{2} \tilde{D} m \omega^{2}}{\lambda^{2}} \sim \frac{M \gamma k T}{\lambda^{2}} m \omega^{2}
$$

The quantity $M \gamma k T / \lambda^{2}$ (divided by time) is a measure of the fluctuations $(\Delta x)_{t h}^{2}$ in the position of the small particle induced by its coupling to the large particle. The quantity $\hbar /(m \omega)$ is representative of the quantum fluctuations $(\Delta x)_{q}^{2}$ of the small particle. For a wide range of choices of the parameters of the model the induced thermal fluctuations are much larger than the quantum fluctuations, and it follows that $\Delta>>\hbar$. (This will always be the case, for example, if the large particle is sufficiently massive). This means, first of all, that as long as $\eta$ is not too small, $w_{Q}[\bar{q}(t)]$ is positive, even though the Wigner function is not, since a smearing of the Wigner function over cells larger in size than about $\hbar$ yields a positive distribution function $[29,28]$.

More importantly, because the smearing is over a cell size very much greater than $\hbar$, an effect essentially the same as decoherence of the small quantum system is produced. To be precise, suppose the initial state of the small quantum system consisted of a superposition of localized wavepackets, e.g., coherent states. Then in the Wigner function, the interference terms between these wavepackets would appear as terms which rapidly oscillate in phase space on a scale the size of $\hbar$. It is well known that smearing the Wigner function over a region much large in size than $\hbar$ strongly suppresses these terms (see Refs.[30,31], for example). Therefore, for all practical purposes we may replace the initial Wigner function with a generally mixed state Wigner function in which the interference terms between wavepackets has been thrown away.

Effectively what is happening here is that the small quantum system alternatives are approximately decoherent because they are approximately correlated with the decohered 
large system alternatives. A similar phenomenon, in the context of quantum measurement theory, was noted by Hartle [24,32].

It could be the case, of course, that the interesting parameters for the model in a particular application are such that (2.9) is not in fact positive. Then the weight function can no longer be interpreted as a probability distribution on $\bar{q}(t)$. However, what one is ultimately interested in is the probability distribution for histories of the large particle, Eq.(2.4), is this is positive by construction, for all choices of parameters.

In this simple linear model, a further simplification may be obtained by inserting (2.9) in (2.5), and carrying out the integration over $\bar{q}(t)$, with the result,

$$
\begin{aligned}
p[\bar{X}(t)]= & \int d p_{0} d q_{0} W_{0}^{B}\left(p_{0}, q_{0}\right) \int \mathcal{D} Q W_{0}^{A}\left(M \dot{Q}_{0}, Q_{0}\right) \exp \left(-\int d t \frac{(Q-\bar{X})^{2}}{\sigma^{2}}\right) \\
& \times \exp \left(-\frac{1}{4 \hbar^{2} \tilde{D}} \int d t\left(M \ddot{Q}+\lambda^{2} \int d t^{\prime} G\left(t, t^{\prime}\right) Q\left(t^{\prime}\right)+\lambda\left[q_{0} \cos \omega t+\frac{p_{0}}{m \omega} \sin \omega t\right]\right)^{2}\right)
\end{aligned}
$$

Loosely speaking, this equation tells us to solve the classical equations of motion for the small particle in terms of their initial data $p_{0}, q_{0}$, and then regard these as stochastic variables with a probability distribution given by a smeared Wigner function.

Another convenient way of writing the weight function (2.5) is

$$
w_{Q}[\bar{q}(t)]=\int \mathcal{D} q \mathcal{W}[q, Q] \exp \left(-\frac{\lambda^{2}}{4 \hbar^{2} \tilde{D} \eta} \int d t(q-\bar{q})^{2}\right)
$$

where

$$
\begin{aligned}
\mathcal{W}[q, Q]=\int \mathcal{D} \xi_{2} & \exp \left(-\frac{i \lambda}{\hbar} \int d t Q \xi_{2}\right) \\
& \times \exp \left(\frac{i}{\hbar} S_{B}\left[q+\frac{1}{2} \xi_{2}\right]-\frac{i}{\hbar} S_{B}\left[q-\frac{1}{2} \xi_{2}\right]\right) \rho_{0}^{B}\left(x_{0}, y_{0}\right)
\end{aligned}
$$

where, recall, $q=\frac{1}{2}(x+y)$ and $\xi_{2}=x-y$, and $S_{B}[x]$ is the free action of the small particle. The quantity $\mathcal{W}[q, Q]$ is the Wigner functional, introduced by Gell-Mann and Hartle [10], 
and is defined, in (2.13), by a functional Wigner transform of the fine-grained decoherence functional for the small particle position histories. It is analagous to the ordinary Wigner function in relation to the density matrix [33]. Hence, in (2.12), we see that the weight function is a smeared Wigner functional. Like the Wigner function, the Wigner functional is not positive in general, but in this case at least, the smeared Wigner functional (2.12) is.

\section{2(C). Recovery of the Naive Semiclassical Approximation}

It is also of interest to examine (2.4) or (2.11) in the limit of very weak coupling, i.e., small $\lambda$. One readily finds, that to lowest order,

$$
\begin{aligned}
p[\bar{X}(t)]= & \int \mathcal{D} Q W_{0}^{A}\left(M \dot{Q}_{0}, Q_{0}\right) \exp \left(-\int d t \frac{(Q-\bar{X})^{2}}{\sigma^{2}}\right) \\
& \times \exp \left(-\frac{1}{4 \hbar^{2} \tilde{D}} \int d t(M \ddot{Q}+\lambda\langle q(t)\rangle)^{2}\right)
\end{aligned}
$$

where

$$
\langle q(t)\rangle=\left\langle q_{0}\right\rangle \cos \omega t+\frac{\left\langle p_{0}\right\rangle}{m \omega} \sin \omega t+\lambda \int d t^{\prime} G\left(t, t^{\prime}\right) Q\left(t^{\prime}\right)
$$

The probability distribution is peaked about the naive semiclassical equation (1.5), and here we see it emerging in the limit of very weak coupling. This is not surprising, since weak coupling corresponds to a very imprecise measurement, and the first thing a very broad measurement sees is the average value.

Although the naive semiclassical approach is very limited, the above considerations show how it might still be useful if used with discretion for certain types of initial states. Note first of all that the expression (2.14), the naive semiclassical result, is not only valid for small $\lambda$. It will also be a valid approximation to (2.11) for initial states whose Wigner 
function is strongly peaked about the mean values of $p$ and $q$, as is the case for a coherent state.

Now consider the key case of an initial state that consists of a superposition of two well-separated phase space localized states, one localized around $p_{1}, q_{1}$, the other localized around $p_{2}, q_{2}$ :

$$
|\Psi\rangle=\alpha_{1}\left|\Psi_{p_{1} q_{1}}\right\rangle+\alpha_{2}\left|\Psi_{p_{2} q_{2}}\right\rangle
$$

Then the Wigner function for this initial state has the form

$$
W=\left|\alpha_{1}\right|^{2} W_{p_{1} q_{1}}+\left|\alpha_{2}\right|^{2} W_{p_{2} q_{2}}+\quad \text { interference terms }
$$

where $W_{p_{1} q_{1}}$ denotes the Wigner function for the state $\left|\Psi_{p_{1} q_{1}}\right\rangle$, and thus is concentrated around $p_{1}, q_{1}$, and similarly for $p_{2}, q_{2}$. Now, the point is that, as we have argued above, the interference terms in the initial Wigner function become highly suppressed as a result of the smearing in Eq.(2.9). Therefore the probability distribution (2.11) has the form

$$
p[\bar{X}(t)] \approx\left|\alpha_{1}\right|^{2} p_{1}[\bar{X}(t)]+\left|\alpha_{2}\right|^{2} p_{2}[\bar{X}(t)]
$$

where $p_{1}[\bar{X}(t)]$ denotes the probability distribution (2.4), but with initial Wigner function $W_{p_{1} q_{1}}$ (for the small system), and similarly for $p_{2}[\bar{X}(t)]$. But these Wigner functions are strongly peaked about their mean values, hence the naive semiclassical expression (2.14) is valid for $p_{1}[\bar{X}(t)]$ and $p_{2}[\bar{X}(t)]$ seperately. The effective description of the coupled classical and quantum system is therefore that the classical system follows the equations of motion

$$
M \ddot{Q}+\lambda\langle q(t)\rangle_{1}=0
$$

with probability $\left|\alpha_{1}\right|^{2}$, and follows

$$
M \ddot{Q}+\lambda\langle q(t)\rangle_{2}=0
$$


with probability $\left|\alpha_{2}\right|^{2}$, where

$$
\langle q(t)\rangle_{1}=\left\langle\Psi_{p_{1} q_{1}}|q(t)| \Psi_{p_{1} q_{1}}\right\rangle
$$

and similarly for $\langle q(t)\rangle_{2}$. This is clearly the intuitively sensible result. Applied undiscerningly, the mean field equations (1.5) would not give this result. Although here we see that, given the small amount of insight provided by decoherence, the mean field equations can be used to good effect.

\section{CONTINUOUS MEASUREMENT THEORY}

The weight function $w_{Q}[\bar{q}(t)]$ turns out to be closely related to continuous measurement theory $[13,14,15,16,17]$. As we have noted, the interaction of the large particle with the small one constitutes a continuous but imprecise measurement of the position of the small particle. In the quantum theory of continuous measurements, the probability for measuring a trajectory $\bar{q}(t)$ up to an imprecision $\sigma_{1}$ is given by the path integral expression,

$$
\begin{aligned}
p[\bar{q}(t)]= & \int \mathcal{D} x \mathcal{D} y \quad \rho_{0}^{B}\left(x_{0}, y_{0}\right) \exp \left(-\int d t \frac{(x-\bar{q})^{2}}{2 \sigma_{1}^{2}}-\int d t \frac{(y-\bar{q})^{2}}{2 \sigma_{1}^{2}}\right) \\
& \times \exp \left(\frac{i}{\hbar} \int d t\left(\frac{1}{2} m \dot{x}^{2}-\frac{1}{2} m \omega^{2} x^{2}-\lambda Q x\right)\right) \\
& \times \exp \left(-\frac{i}{\hbar} \int d t\left(\frac{1}{2} m \dot{y}^{2}-\frac{1}{2} m \omega^{2} y^{2}-\lambda Q y\right)\right)
\end{aligned}
$$

As above, introduce the variables $q=\frac{1}{2}(x+y), \xi=x-y$, hence,

$$
\begin{aligned}
p[\bar{q}(t)]= & \int \mathcal{D} q \mathcal{D} \xi \quad \rho_{0}^{B}\left(q_{0}+\frac{1}{2} \xi_{0}, q_{0}-\frac{1}{2} \xi_{0}\right) \exp \left(-\int d t \frac{(q-\bar{q})^{2}}{\sigma_{1}^{2}}-\int d t \frac{\xi^{2}}{4 \sigma_{1}^{2}}\right) \\
& \times \exp \left(-\frac{i}{\hbar} \int d t \xi\left(m \ddot{q}+m \omega^{2} q+\lambda Q\right)-\frac{i}{\hbar} m \dot{q}(0) \xi(0)\right)
\end{aligned}
$$


Comparing with the expression for the weight function (2.5), we see that it is very similar, if $\sigma_{1}$, which is so far arbitrary, is taken to be,

$$
\sigma_{1}^{2}=\frac{4 \hbar^{2} \tilde{D} \eta}{\lambda^{2}} \sim \frac{M \gamma k T}{\lambda^{2}}
$$

Although note that (3.1), (3.2) differ from (2.5) by the absence of the term

$$
\exp \left(-\int d t \frac{\xi^{2}}{4 \sigma_{1}^{2}}\right)
$$

in (2.5). Hence Eq.(2.5) is not exactly the same as the continuous measurement formula (3.1). However, we will see that they are very close.

Carrying out the $\xi$ integration we obtain,

$$
\begin{aligned}
p[\bar{q}(t)] & =\int \mathcal{D} q W_{0}^{B}\left(m \dot{q}_{0}, q_{0}\right) \\
& \times \exp \left(-\int d t \frac{(q-\bar{q})^{2}}{\sigma_{1}^{2}}-\frac{\sigma_{1}^{2}}{\hbar^{2}} \int d t\left(m \ddot{q}+m \omega^{2} q+\lambda Q\right)^{2}\right)
\end{aligned}
$$

With the above choice of $\sigma_{1}$,

$$
\frac{\sigma_{1}^{2}}{\hbar^{2}} \sim \frac{M \gamma k T}{\hbar^{2} \lambda^{2}}
$$

For macroscopic values of $M, \gamma$ and $T$, and assuming $\lambda^{2}$ is not unusually large, the factor of $\hbar^{2}$ in the denominator ensures that $\sigma_{1}^{2} / \hbar^{2}$ is very large. The second exponential in (3.5) is therefore very close to a delta function, Eq.(3.5) is therefore very close in form to the alternative expression for the weight function (2.7).

Note that the width $\sigma_{1}$ of the effective "measurement" of $q$ depends on two things. First of all it depends on the coupling $\lambda$ and is smaller the larger $\lambda$ is, corresponding to the notion that stronger interactions produce more precise measurements. Secondly, it depends on the combination $M \gamma k T$, which is a measure of the thermal fluctuations endured by the large particle as a result of its interaction with the environment, and hence, is a 
measure of the precision to within which the trajectory of the large particle is defined. $\sigma_{1}$ increases with increasing $M \gamma k T$, which is to be expected, since the precision with which the large particle can measure the small particle depends on the precision with which the large particle's properties are themselves defined.

Given the close resemblance to continuous quantum measurement theory, the question remains, why did we not get exactly the formula for continuous quantum measurements? After all, in the decoherent histories approach it is possible to derive standard quantum measurement theory (i.e., measurements represented by exact projection operators at discrete moments of time), under certain idealized conditions [24,32]. The answer to this is probably to be found in the nature of the simple model we are considering and in particular, the couplings between the subsystems. For example, in the model considered here, we coupled $x$ to just a single degree of freedom $X$ (in turn coupled to the environment). Coupling to a large number of degrees of freedom may lead to the missing factor, (3.4), in the same way that the coupling of $X$ to a large environment produces the factor involving $\int d t(X-Y)^{2}$ in Eq.(2.1). Hence it is quite possible that a closer connection between decoherent histories and continuous quantum measurements might be found by exploring more general types of models couplings. This is tangential to the main theme of this paper, so will be explored elsewhere.

It is perhaps of interest to note that the above result on continuous measurement can be re-expressed in terms of evolution equations, and this in fact casts our results in a form very close to the original mean field equations (1.5) [18]. For a pure initial state, the probability formula for continuous meausrements (3.1) has the form $\left\langle\Psi_{\bar{q}} \mid \Psi_{\bar{q}}\right\rangle$, for a wave function $\Psi_{\bar{q}}$, whose path integral representation is given by "half" of (3.1). From this one can define a normalized state $|\psi\rangle$ whose time evolution is given by the non-linear stochastic 
equation,

$$
\frac{d}{d t}|\psi\rangle=\left(-\frac{i}{\hbar} H-\frac{1}{\sigma_{1}^{2}}(\hat{q}-\langle q\rangle)^{2}\right)|\psi\rangle+\frac{1}{\sigma_{1}}(\hat{q}-\langle q\rangle)|\psi\rangle \eta(t)
$$

Here $\eta(t)$ is Gaussian white noise whose linear and quadratic means are,

$$
\langle\eta(t)\rangle_{S}=0, \quad\left\langle\eta(t) \eta\left(t^{\prime}\right)\right\rangle_{S}=\delta\left(t-t^{\prime}\right)
$$

where \langle\rangle$_{S}$ denotes stochastic averaging. $\eta$ is related to the measured variable $\bar{q}$ by

$$
\bar{q}=\langle\psi|\hat{q}| \psi\rangle+\frac{1}{2} \sigma_{1} \eta(t)
$$

$H$ is the Hamiltonian for the small system (in this case a harmonic oscillator, with $X(t)$ as an external source).

Hence, to the extent that the semiclassical equations we have derived are equivalent to continuous quantum measurement, the new equations that replace the mean field equations (1.5) are

$$
M \ddot{Q}+\lambda\langle\psi|q| \psi\rangle+\frac{1}{2} \lambda \sigma_{1} \eta(t)=0
$$

where $|\psi\rangle$ evolves according to the stochastic non-linear equation (3.7). Note that with the value of $\sigma_{1}$ is given by (3.3), the noise term $\lambda \sigma_{1} \eta(t)$ is independent of $\lambda$, so remains as $\lambda \rightarrow 0$, and describes the thermal fluctuations of the large particle.

The noise term describes fluctuations about the mean. This sort of modification, in the context of (1.1), has been considered before [5,34]. More significant is the fact the state evolves according to (3.7), and it is the properties of this equation that correspond to the separation of initial superposition states described in Section 2(C) [18].

The above scheme was put forward in Ref.[18] as a phenomenological model for the coupling of classical and quantum variables, and the value of $\sigma_{1}$ proposed there on general physical grounds agrees with the one derived here. 


\section{NON-LINEAR COUPLINGS}

Section 2 concentrated entirely on the case of a free particle linearly coupled to a harmonic oscillator. Now we show how these considerations can be extended to more complicated cases. First we consider the case of a particle in a potential $V(X)$ coupled to a harmonic oscillator via a coupling of the form, $g(X) x$. The decoherence functional (2.1) is therefore replaced by

$$
\begin{aligned}
D[\bar{X}, \bar{Y}]= & \int \mathcal{D} X \mathcal{D} Y \mathcal{D} x \mathcal{D} y \rho_{0}^{A}\left(X_{0}, Y_{0}\right) \rho_{0}^{B}\left(x_{0}, y_{0}\right) \\
& \times \exp \left(-\int d t \frac{(X-\bar{X})^{2}}{2 \sigma^{2}}-\int d t \frac{(Y-\bar{Y})^{2}}{2 \sigma^{2}}\right) \\
& \times \exp \left(\frac{i}{\hbar} \int d t\left(\frac{1}{2} M \dot{X}^{2}-V(X)-\frac{1}{2} M \dot{Y}^{2}+V(Y)\right)-D \int d t(X-Y)^{2}\right) \\
& \times \exp \left(\frac{i}{\hbar} \int d t\left(\frac{1}{2} m \dot{x}^{2}-\frac{1}{2} m \omega^{2} x^{2}-g(X) x\right)\right) \\
& \times \exp \left(-\frac{i}{\hbar} \int d t\left(\frac{1}{2} m \dot{y}^{2}-\frac{1}{2} m \omega^{2} y^{2}-g(Y) y\right)\right)
\end{aligned}
$$

This case is actually handled quite simply using the fact that the integration over $X$ and $Y$ is strongly concentrated around $X=Y$. As before, write $X=Q+\frac{1}{2} \xi_{1}$ and $Y=Q-\frac{1}{2} \xi_{1}$. Then we have

$$
\begin{aligned}
V(X)-V(Y) & =\xi_{1} V^{\prime}(Q)+O\left(\xi_{1}^{3}\right) \\
g(X) x-g(Y) y & =g(Q)(x-y)+\frac{1}{2} \xi_{1} g^{\prime}(Q)(x+y)+O\left(\xi_{1}^{2}\right)
\end{aligned}
$$

The $\xi_{1}$ integral is readily done, and we obtain for the probabilities,

$$
\begin{aligned}
p[\bar{X}(t)]= & \int \mathcal{D} Q \mathcal{D} x \mathcal{D} y W_{0}^{A}\left(M \dot{Q}_{0}, Q_{0}\right) \rho^{B}\left(x_{0}, y_{0}\right) \\
& \times \exp \left(-\int d t \frac{(Q-\bar{X})^{2}}{\sigma^{2}}-\frac{1}{4 \hbar^{2} \tilde{D}} \int d t\left(M \ddot{Q}+V^{\prime}(Q)+\frac{1}{2} g^{\prime}(Q)(x+y)\right)^{2}\right) \\
& \times \exp \left(\frac{i}{\hbar} \int d t\left(\frac{1}{2} m \dot{x}^{2}-\frac{1}{2} m \omega^{2} x^{2}-g(Q) x\right)\right)
\end{aligned}
$$




$$
\times \exp \left(-\frac{i}{\hbar} \int d t\left(\frac{1}{2} m \dot{y}^{2}-\frac{1}{2} m \omega^{2} y^{2}-g(Q) y\right)\right)
$$

This may be written

$$
\begin{aligned}
p[\bar{X}(t)] & =\int \mathcal{D} Q \mathcal{D} \bar{q} W_{0}^{A}\left(M \dot{Q}_{0}, Q_{0}\right) w_{Q}[\bar{q}(t)] \\
& \times \exp \left(-\int d t \frac{(Q-\bar{X})^{2}}{\sigma^{2}}-\frac{1}{4 \hbar^{2} \tilde{D}(1-\eta)} \int d t\left(M \ddot{Q}+V^{\prime}(Q)+g^{\prime}(Q) \bar{q}\right)^{2}\right)
\end{aligned}
$$

where

$$
\begin{aligned}
w_{Q}[\bar{q}(t)]= & \int \mathcal{D} x \mathcal{D} y \rho_{0}^{B}\left(x_{0}, y_{0}\right) \exp \left(-\frac{1}{4 \hbar^{2} \tilde{D} \eta} \int d t\left(g^{\prime}(Q)\right)^{2}\left(\frac{(x+y)}{2}-\bar{q}\right)^{2}\right) \\
& \times \exp \left(\frac{i}{\hbar} \int d t\left(\frac{1}{2} m \dot{x}^{2}-\frac{1}{2} m \omega^{2} x^{2}-g(Q) x\right)\right) \\
& \times \exp \left(-\frac{i}{\hbar} \int d t\left(\frac{1}{2} m \dot{y}^{2}-\frac{1}{2} m \omega^{2} y^{2}-g(Q) y\right)\right)
\end{aligned}
$$

Introducing $q=\frac{1}{2}(x+y)$ and $\xi_{2}=x-y$, the $\xi_{2}$ integral may be done with the result,

$$
\begin{aligned}
w_{Q}[\bar{q}(t)]= & \int \mathcal{D} q W_{0}^{B}\left(m \dot{q}_{0}, q_{0}\right) \exp \left(-\frac{1}{4 \hbar^{2} \tilde{D} \eta} \int d t\left(g^{\prime}(Q)\right)^{2}(q-\bar{q})^{2}\right) \\
& \times \delta\left[m \ddot{q}+m \omega^{2} q+g(Q)\right]
\end{aligned}
$$

This is very similar to the continuous measurement formula (3.1), (3.2) if we allow the imprecision parameter $\sigma_{1}$ to depend on the external field.

The next more complicated case we consider is that in which the coupling between the particles is of the form $g(X) f(x)$. It is straightforward to show that the probability is then

$$
\begin{aligned}
p[\bar{X}(t)]= & \int \mathcal{D} Q \mathcal{D} x \mathcal{D} y W_{0}^{A}\left(M \dot{Q}_{0}, Q_{0}\right) \rho^{B}\left(x_{0}, y_{0}\right) \\
& \times \exp \left(-\int d t \frac{(Q-\bar{X})^{2}}{\sigma^{2}}-\frac{1}{4 \hbar^{2} \tilde{D}} \int d t\left(M \ddot{Q}+V^{\prime}(Q)+\frac{1}{2} g^{\prime}(Q)(f(x)+f(y))\right)^{2}\right) \\
& \times \exp \left(\frac{i}{\hbar} \int d t\left(\frac{1}{2} m \dot{x}^{2}-\frac{1}{2} m \omega^{2} x^{2}-g(Q) f(x)\right)\right) \\
& \times \exp \left(-\frac{i}{\hbar} \int d t\left(\frac{1}{2} m \dot{y}^{2}-\frac{1}{2} m \omega^{2} y^{2}-g(Q) f(y)\right)\right)
\end{aligned}
$$


Again this may be cast in the form

$$
\begin{aligned}
p[\bar{X}(t)] & =\int \mathcal{D} Q \mathcal{D} \bar{f} W_{0}^{A}\left(M \dot{Q}_{0}, Q_{0}\right) w_{Q}[\bar{f}(t)] \\
& \times \exp \left(-\int d t \frac{(Q-\bar{X})^{2}}{\sigma^{2}}-\frac{1}{4 \hbar^{2} \tilde{D}(1-\eta)} \int d t\left(M \ddot{Q}+V^{\prime}(Q)+g^{\prime}(Q) \bar{f}\right)^{2}\right)
\end{aligned}
$$

where

$$
\begin{aligned}
w_{Q}[\bar{f}(t)]= & \int \mathcal{D} x \mathcal{D} y \rho_{0}^{B}\left(x_{0}, y_{0}\right) \exp \left(-\frac{1}{4 \hbar^{2} \tilde{D} \eta} \int d t\left(g^{\prime}(Q)\right)^{2}\left(\frac{(f(x)+f(y))}{2}-\bar{f}\right)^{2}\right) \\
& \times \exp \left(\frac{i}{\hbar} \int d t\left(\frac{1}{2} m \dot{x}^{2}-\frac{1}{2} m \omega^{2} x^{2}-g(Q) f(x)\right)\right) \\
& \times \exp \left(-\frac{i}{\hbar} \int d t\left(\frac{1}{2} m \dot{y}^{2}-\frac{1}{2} m \omega^{2} y^{2}-g(Q) f(y)\right)\right)
\end{aligned}
$$

Again it is similar to the continuous measurement formula, but now the variable being measure is not position $x$, but $f(x)$, as one would expect since it is this that couples to the large particle. It is not possible to evaluate $w_{Q}$ any further in this case.

Overall, therefore, although the evaluation of the path integrals is less explicit in these more complicated case, the general pattern is the same as in the linear case described in Section 2: the large particle follows near-deterministic equations of motion but with a stochastic forcing term due the small particle, whose probability distribution bears a close resemblance to the formula of continuous quantum measurement theory.

\section{COUPLING TO ENERGY}

Another case of particular interest, especially in connection with the semiclassical Einstein equations (1.1), is the case in which the large particle couples to the energy of the small particle. The considerations of the previous sections apply to this case very easily. In fact, this case turns out to be somewhat simpler. 
Let the Hamiltonian of the total closed system, including large system (A), small system (B) and environment (E), be

$$
H=H_{A}+H_{A B}+H_{\mathcal{E}}+H_{A \mathcal{E}}
$$

where

$$
H_{A B}=\lambda g(X) h
$$

and $h$ is a harmonic oscillator Hamiltonian. $g(X)$ is an arbitrary function of $X . H_{A}, H_{\mathcal{E}}$ and $H_{A \mathcal{E}}$ are as before. Let the initial state of the small system be written in terms of energy eigenfunctions,

$$
\rho^{B}=\sum_{E E^{\prime}} \rho_{E E^{\prime}}|E\rangle\left\langle E^{\prime}\right|
$$

where $h|E\rangle=E|E\rangle$. Then, because $h$ commutes with everything, whenever $H$ operates on $|E\rangle, h$ is replaced by the eigenfunction $E$.

It is then straightforward to see that the probabilities for histories of the large particle are

$$
\begin{aligned}
p[\bar{X}(t)]= & \sum_{E} \rho_{E E} \int \mathcal{D} X \mathcal{D} Y \rho_{0}^{A}\left(X_{0}, Y_{0}\right) \\
& \times \exp \left(-\int d t \frac{(X-\bar{X})^{2}}{2 \sigma^{2}}-\int d t \frac{(Y-\bar{X})^{2}}{2 \sigma^{2}}\right) \\
& \times \exp \left(\frac{i}{\hbar} \int d t\left(\frac{1}{2} M \dot{X}^{2}-\frac{1}{2} M \dot{Y}^{2}\right)-D \int d t(X-Y)^{2}\right) \\
& \times \exp \left(-\frac{i}{\hbar} \int d t(g(X)-g(Y)) E\right)
\end{aligned}
$$

The summation over $E$ and $E^{\prime}$ is diagonal because $h$ commutes with everything else so the states $|E\rangle$ are preserved under evolution by the total Hamiltonian, and the trace in the decoherence functional then contains the term $\left\langle E \mid E^{\prime}\right\rangle$. 
Introducing $Q$ and $\xi$ as before, this becomes,

$$
\begin{aligned}
p[\bar{X}(t)]= & \sum_{E} \rho_{E E} \int \mathcal{D} Q W_{0}^{A}\left(M \dot{Q}_{0}, Q_{0}\right) \\
& \times \exp \left(-\int d t \frac{(Q-\bar{X})^{2}}{\sigma^{2}}-\frac{1}{4 \hbar^{2} \tilde{D}} \int d t\left(M \ddot{Q}+g^{\prime}(Q) E\right)^{2}\right)
\end{aligned}
$$

It is straightforward to then rewrite this in terms of continuous imprecise measurement of energy. For simplicity take $g(Q)=\lambda Q$, then

$$
\begin{aligned}
p[\bar{X}(t)]= & \int d \bar{E} \int \mathcal{D} Q W_{0}^{A}\left(M \dot{Q}_{0}, Q_{0}\right) w(\bar{E}) \\
& \times \exp \left(-\int d t \frac{(Q-\bar{X})^{2}}{\sigma^{2}}-\frac{1}{4 \hbar^{2} \tilde{D}(1-\eta)} \int d t(M \ddot{Q}+\lambda \bar{E})^{2}\right)
\end{aligned}
$$

where

$$
w(\bar{E})=\sum_{E} \rho_{E E} \exp \left(-\frac{\lambda^{2} \tau}{4 \hbar^{2} \tilde{D} \eta}(E-\bar{E})\right)
$$

where $\tau$ is the time duration of the histories. Eq.(5.7) is the formula for the continous imprecise measurement of energy, using Gaussian projectors, to a width of order $\hbar^{2} \tilde{D} /\left(\lambda^{2} \tau\right)$. Here the connection with continous measurements is precise, although this is clearly due to the simplicity of this particular case.

Of perhaps greater interest is the situation in which the energy of the small particle couples in a non-trivial way to the large particle, for example, through a small particle Hamiltonian of the form

$$
H=f(X) p^{2}+g(X) x^{2}
$$

This is a more realistic model of the way in which matter couples to gravity (for example, in cosmology, a single mode of a scalar field coupled to the scale factor). This is much more complicated to deal with and will be treated elsewhere. 


\section{DISCUSSION}

We have derived the form of the effective equations of motion for some simple systems consisting of a large particle coupled to a small particle, and coupled also to a thermal environment in order to produce the decoherence necessary for classicality of the large particle. The resultant effective theory has the form of a classical variable coupled to a stochastic variable $\bar{x}(t)$, where the probability distribution for the stochastic variable is given by a certain weight function (most generally, Eq. (4.9)). This weight function is closely related (although not exactly the same) as the probability for continous imprecise measurements of the position of the small particle. In the case of coupling to energy, it is exactly the same as the continous measurement theory result.

The weight function has the property that it suppresses the interference between localized wave packets for the small particle. Hence one of the more unsatisfactory features of the naive semiclassical approximation is avoided, and the intuitively sensible result that localized wave packet initial states may be treated separately is restored.

The derived semiclassical theory suggests the form of a possible semiclassical theory even when the quantum theory of the variables that are taken to be classical is not known. It is the following: in the equations of motion for the classical system, which involves a coupling to the quantum system, replace the quantum variables with stochastic variables whose probabilities are given by a weight function of the form (2.5) (or its generalizations). The classical variable $Q$ is regarded as an external classicl source in (2.5) and the path integral is well-defined, even if the quantum theory of $Q$ is not known.

The only aspect of (2.5) that was inherited from the quantum theory of the classical variables is the width of the Gaussian, $\hbar^{2} \tilde{D}^{2} / \lambda^{2}$. However, we can see from (2.4) that

physically, the factor $\hbar^{2} \tilde{D}$ is a measure of the precision to within which the trajectories of 
classical variables are defined, and we can imagine that this number could be determined (or at least bounded) by experiment.

A very similar semiclassical scheme (using the continous measurement formula) was described in Ref.[18], and the results of this paper give partial substantiations of that scheme.

We have used the decoherent histories approach to derive effective field equations, for reasons stated in Section 1. It is, however, quite possible that other approaches to emergent classicality may be used, such as the density matrix approach $[35,31]$, the quantum state diffusion picture $[36,37,22]$, or the hybrid representation of composite quantum systems $[38,39,40]$. A system similar to that considered in this paper has been analyzed in the

quantum state diffusion picture by Zoupas [41], and a simple spin system by $\mathrm{Yu}$ and Zoupas [42].

\section{ACKNOWLEDGEMENTS}

I am very grateful to Lajos Diósi and Jason Twamley for useful conversations.

\section{REFERENCES}

1. L.Rosenfeld, Nucl. Phys. 40, 353 (1963).

2. C.Moller, in Les Theories Relativistes de la Gravitation, edited by A.Lichnerowicz and M.A.Tonnelat (CNRS, Paris, 1962).

3. L.H.Ford, Ann. Phys. (N.Y.) 144, 238 (1982). 
4. J.B.Hartle and G.T.Horowitz, Phys. Rev. D24, 257 (1981).

5. C-I.Kuo and L.H.Ford, Phys. Rev. D47, 4510 (1993).

6. D.N.Page and C.D.Geilker, Phys. Rev. Lett. 47, 979 (1981).

7. T.W.B.Kibble, in Quantum Gravity 2: A Second Oxford Symposium, edited by C.J.Isham, R.Penrose and D.W.Sciama (Oxford University Press, New York, 1981).

8. I.V.Aleksandrov, Z.Naturf. 36A, 902 (1981); A.Anderson, Phys. Rev. Lett. 74, 621 (1995); Phys. Rev. Lett. 76, 4090 (1996); W.Boucher and J.Traschen, Phys. Rev. D37, 3522 (1988); K.R.W.Jones, Phys. Rev. Lett. 76, 4087 (1996); L.Diósi, Phys. Rev. Lett. 76, 4088 (1996); I.R.Senitzky, Phys. Rev. Lett. 76, 4089 (1996).

9. M.Gell-Mann and J.B.Hartle, in Complexity, Entropy and the Physics of Information, SFI Studies in the Sciences of Complexity, Vol. VIII, W. Zurek (ed.) (Addison Wesley, Reading, 1990); and in Proceedings of the Third International Symposium on the Foundations of Quantum Mechanics in the Light of New Technology, S. Kobayashi, H. Ezawa, Y. Murayama and S. Nomura (eds.) (Physical Society of Japan, Tokyo, 1990).

10. M.Gell-Mann and J.B.Hartle, Phys.Rev. D47, 3345 (1993).

11. R.Griffiths, J.Stat.Phys. 36, 219 (1984).

12. R.Omnès, The Interpretation of Quantum Mechanics (Princeton University Press, Princeton, 1994); Rev.Mod.Phys. 64, 339 (1992), and references therein.

13. A.Barchielli, L.Lanz and G.M.Prosperi, Il Nuovo Cimento 72B, 79 (1982).

14. V.P.Belavkin and P.Staszewski, Phys.Rev. A45, 1347 (1992). 
15. C.M.Caves and G.J.Milburn, Phys.Rev. A36, 5543 (1987).

16. L.Diósi, Phys.Rev. A42, 5086 (1990).

17. L.Diósi, Phys.Lett. 129A, 419 (1988).

18. L.Diósi and J.J.Halliwell,, "Coupling Classical and Quantum Variables using Continous Quantum Measurement Theory", Imperial College preprint 96-97/46, quantph/9705XX (1997).

19. J.J.Halliwell, "A Review of the Decoherent Histories Approach to Quantum Mechanics", in Fundamental Problems in Quantum Theory, edited by D.Greenberger and A.Zeilinger, Annals of the New York Academy of Sciences, Vol 775, 726 (1994).

20. J.B.Hartle, in, Proceedings of the Cornelius Lanczos International Centenary Confererence, edited by J.D.Brown, M.T.Chu, D.C.Ellison and R.J.Plemmons (SIAM, Philadelphia, 1994)

21. J.J.Halliwell, Phys.Rev. D48, 4785 (1993).

22. J.J.Halliwell and A.Zoupas, Phys.Rev. D52, 7294 (1995); "Post-decoherence density matrix propagator for quantum Brownian motion", IC preprint 95-96/67, quantph/9608046 (1996), accepted for publication in Phys.Rev.D (1997).

23. H.F.Dowker and J.J.Halliwell, Phys. Rev. D46, 1580 (1992).

24. J.B.Hartle, in Quantum Cosmology and Baby Universes, S. Coleman, J. Hartle, T. Piran and S. Weinberg (eds.) (World Scientific, Singapore, 1991).

25. J.J.Halliwell, "Aspects of the Decoherent Histories Approach to Quantum Theory", in Stochastic Evolution of Quantum States in Open Systems and Measurement Processes, edited by L.Diósi, L. and B.Lukács (World Scientific, Singapore, 1994). 
26. R.P.Feynman and F.L.Vernon, Ann. Phys. (N.Y.) 24, 118 (1963).

27. A.O.Caldeira and A.J.Leggett, Physica 121A, 587 (1983).

28. J.J.Halliwell, Phys.Rev. D46, 1610 (1992).

29. K.Husimi, Proc.Phys.Math.Soc. Japan 22, 264 (1940).

30. J.P.Paz, S.Habib and W.Zurek, Phys. Rev. D47, 488 (1993).

31. W. Zurek, Prog.Theor.Phys. 89, 281 (1993); Physics Today 40, 36 (1991); in, Physical Origins of Time Asymmetry, edited by J.J.Halliwell, J.Perez-Mercader and W.Zurek (Cambridge University Press, Cambridge, 1994).

32. J.B.Hartle, in Proceedings of the 1992 Les Houches Summer School, Gravitation et Quantifications, edited by B.Julia and J.Zinn-Justin (Elsevier Science B.V., 1995)

33. N.Balazs and B.K.Jennings, Phys. Rep. 104, 347 (1984), M.Hillery, R.F.O'Connell, M.O.Scully and E.P.Wigner, Phys. Rep. 106, 121 (1984); V.I.Tatarskii, Sov.Phys.Usp 26, 311 (1983).

34. B.L.Hu and A.Matacz, Phys. Rev. D51, 1577 (1995).

35. E.Joos and H.D.Zeh, Zeit.Phys. B59, 223 (1985).

36. N. Gisin and I.C.Percival, J.Phys. A26, 2233 (1993); A26, 2245 (1993).

37. I.C.Percival, J.Phys. A27, 1003 (1994).

38. L.Diósi, "A True Equation to Couple Classical and Quantum Variables", preprint quant-ph/9510028 (1995),

39. L.Diósi, Quantum Semiclass.Opt. 8, 309 (1996). 
40. L.Diósi, preprint quant-ph/9610037 (1996). To appear in Fundamental Problems in Quantum Physics, edited by M.Ferrero and A. van der Merwe (Kluwer, Denver, 1997).

41. A.Zoupas, "Coupling of Quantum to Classical in the Presence of a Decohering Environment", Imperial College preprint (1997).

42. T.Yu and A.Zoupas, in preparation. 